\title{
Secure Social Media Spaces for Communities of Vulnerable People
}

\author{
Elias Pimenidis \\ Department of Computer Science and Creative Technologies \\ University of the West of England \\ Bristol, United Kingdom \\ elias.pimenidis@uwe.ac.uk
}

\author{
Nikolaos Polatidis \\ School of Computing, Engineering and Mathematics \\ University of Brighton \\ Brighton, United Kingdom \\ n.polatidis@brighton.ac.uk
}

\begin{abstract}
This paper presents a proposal for developing secure social media spaces for groups of people that could be vulnerable to external influences. These could be refuges from war torn or politically unstable countries that could be persecuted by political opponents, or young children that could be targeted online by paedophiles and other online criminals. The system utilizes Block-Chaining technologies to provide the secure environment and Recommender Systems to link members to sub-groups based on their preferable discussion topics and ideas. There are challenges in the proposal as the key issue of trust in the system must be guaranteed by a third party. This organization will need to verify the identity of every subscriber and then create secure user identities / profiles that guarantee the anonymity of members. Block-chaining technologies will also allow the monitoring of user behaviour to ensure the trustworthiness of the environment is maintained. The cost of the system and its operation will be another consideration. The reputation of Block-Chaining technologies due to its links with crypto-currencies should not be considered a negative factor as there are many successful implementations in everyday life
\end{abstract}

Keywords-Social Media, Trust, Secure Spaces, Recommender Systems, Blockchain Technology

\section{INTRODUCTION}

The dawn of the twenty first century has witnessed unprecedented destruction of life and uprooting of millions of people, often leading to mass migration. World and totalitarian regimes have contributed to the destruction of democracy in a lot of countries where peace prevailed before and citizens were normally involved in democratic processes. By leaving their place of domicile among other important material belonging and cultural activities migrants are mostly deprived of their political activities and their opportunity in contributing to the democratic processes there. Human nature frequently leads migrants to long for the return to their land of origin and any reluctance to do so is based on insecurity as to the economic and democratic environments upon their return [1]. The former is beyond the scope of this work, but the latter is where this research focuses upon. The authors believe that given the opportunity to participate in the rebuilding of the democratic environment from a distance any fears of insecurity may be overcome and the valuable input of refugees or migrants can contribute to faster restoration of democracy in their countries of origin [2]. The objective of this work is not to explore the political implications and intricacies of such effort, but to introduce the concept and the research of the technical support and infrastructure that would provide secure environments.

Trust can be cultivated in humans and internet users can be convinced to trust the systems they work with if the integrity of the procedures and their privacy as users are guaranteed [3]. To this effect the authors present their concept of combining the use of intelligent algorithms of the type used in recommender systems in combination with Blockchaining to enhance the privacy of social media. In doing so frequent users of social media can feel more relaxed in using them as fora for political debates, online political meetings and decision-making processes that could have an impact on restoring democracy in troubled countries while allowing all people with the right to do so to be involved.

\section{DEMOCRACY AND Displaced PEOPLE}

At present the United Nations confirm that the world is experiencing a major crisis with a record number of displaced people, often fleeing war, oppressive regimes and poverty [4]. The role of this work is not to engage in a political agenda as to the causes of such misfortunes, but to introduce and further explore the use of web-based services and the role social media can play to engage displaced people with news the political developments in their home countries and involve them in a digital democratic process.

In Europe alone the arrival of refugees has been recorded in millions of people in the past five years alone. As efforts to support, feed, shelter and provide a sense of normality in life for all these people have proved a daily struggle, they cannot be the focus of our research but hopefully could benefit by the outcome of such work in the near future.

Other research has documented the trail paths of refugees, how they communicate, the risks they undergo and their dreams versus the heavy human cost in lives lost, or lives destroyed by separation [5, $2 \& 6]$. This work focuses on how trust can be gained and utilized in the sphere of social media and how advanced technologies can help cultivate this trust by providing secure and trusted political forums to serve the needs of displaced people.

In recent cases of refugee movements large masses of people have either been trapped in refugee camps in countries along the shores of the Mediterranean Sea or have been relocated to some of Europe's more affluent countries $[8,9]$. Looking at the data that maps the origins of these people on can clearly see the patterns of countries where long and strong struggles for Democracy have often led to prolonged internal conflicts. Despite the evil that has driven them from their homes, many particularly the young believe that there is a future in their countries. Despite having relocated to more peaceful places where freedom of speech and democratic rights is the norm, they are willing to contribute to the restoration of Democracy and normal life in their countries [9].

Despite their willingness to rebuild though most if not all refugees and displaced people do not trust the official media in their country of origin. These, either due to the conflict situation or due to being controlled by one or the other side, provide skewed, biased and often rather dated information. Thus the people build relationships within the world of social media and use them as their basis for communication and interaction with the world they left behind [5]. Social Media often becomes their "home for Democracy" their ability to debate issues (from a distance) and show that they care; they have a voice and commit their will to participate for a better future [7]. One can assume that the distance alone can give 
displaced people a sense of security and gain their trust. Over social media they can express their views freely, they can lay down plans and sometimes they can expose ill-practice that suppresses democratic values in their homelands. All this might sound reasonable, but often the situation is not as simple. Families are quite frequently split across more than one location and even different countries [8]. In the difficult circumstances of war-torn lands the risk of repercussions against one person's extended families due to political reasons is quite high. Thus the use of social media needs to cater for privacy if it is to gain momentum into a tool in promoting and rebuilding democracy.

\section{Prepare Your PAPER Before Styling}

The field of social media and its relationship with trust, the risk of such trust being betrayed and often such betrayal leading to disaster are not new and have been researched quite thoroughly from the point of view of social scientists in particular.

In general social media are not anonymous, and social cues are mostly visible, because they allow for the exchange of text as well as photographs and other media files. Two of the most popular such platforms, Facebook, on which more than $70 \%$ of adult, American, internet users participate, and Twitter, used by more than $23 \%$ of the adult, internet population subscribes to have billions of users between them. Using such systems and sharing information in a largely open environment entails a level of risk. Risks though are perceived differently by different groups of users and the level of risk each type of user is prepared to take depends on a wide range of issues [7, 10].

Raine and Duggan [11] claim that Americans are keener to provide personal information over the internet depending on any rewards offered. On the other hand, social media for some groups of people are a means of survival.

For Somali refugees Facebook is the most popular communication tool. As a media platform, it allows users to keep in touch with childhood friends with whom they lost contact when they left their homeland, with friends living in Europe and other parts of the world and with those individuals with whom friendships were formed while in exile [5]. Such contacts are valuable and very sensitive to them. They cannot afford to expose them to any risks and therefore they will be very careful as to what kind of contacts they maintain with the external world if they are to limit the risks they expose those close and dear to them. Dincelli and Goel [12] claim that different people with the same level of knowledge and same background behave differently in ensuring their privacy online. Desire for privacy varies considerably based on different factors; for instance, certain cultures tend to have stronger desire for privacy. Their research focused on exploring whether culture influence users' attitude and behaviour on privacy protection. They propose that adequate precautions via tailored security controls, support and privacy policies for different countries/cultures might aid to reduce the number of security and privacy incidents online. This appears to be a valid proposition and one functioning within the confines of settled and secure democratic society expects that this is the case. In the situation refugees though it is quite difficult to gain that level of trust as the risk to which their views and information are exposed are usually away from their control and may affect people they cannot protect. This is confirmed by the research carried out by Trepte and Masur [13] who confirm that people might perceive different types of information as differing in the extent to which they affect privacy levels. This is closely related to cultural differences, places of origin and life experiences that force people to be more or less sensitive to certain types of information.

Politics and governments in particular are continuously increasing their involvement with social media nowadays. In the western world in particular governments are often embarking in projects that aim at attracting attention and participation from citizens in attempts to improve services, engage more citizens in the decision-making process and further develop a citizen centric sense of democracy $[10,14$ and 15]. Such efforts meet with varying degrees of success though. A variety of reasons have been cited. Some citizens do not trust this direct contact with their governments at personal level, while others are mostly indifferent as the wealth of publicly available information does not necessitate the need for such closer engagement. Another reason where people in stable democratic countries might feel they need to distance themselves from interacting with government through social media is that fact that a lot of social network sites link the contextual presence with its physical location and thus introduce an eerie sense of excessive control. Thus a lot of social media users are associated with lower willingness to discuss a political issue in person, across a range of settings, from the private home, to public meetings. In some contexts, particularly the workplace and this contributes to a spiral of silence that can further hinder political conversation [7].

For a lot of social media users there are issues of privacy concerns and these are in a sense justified due to the open nature of some of the most predominant social networks; this is particularly true where friends of friends can see the information and material a user may share. This becomes even more challenging where political views are shared. Most social media users would be categorized in the following four classes of areas of concern:

- Fear of information misuse,

- Fear that others might find my private information,

- Fear that others might use my private information, and

- $\quad$ use of private information is unforeseen [16].

Earlier in this section there was reference to the efforts by western democracies to provide inclusive government through their online presence. Hamajoda [17] discusses the need for parliaments in Western African republics to strengthen the communication channels between lawmakers, the electorate and government agencies. The research cites the emergence of and power of telecommunications and digital media that could expose and damage the links between governments and citizens. The need to expand to advanced online services and enhance the participation of citizens to online decision-making while at the same time politicians need to be trained and change in their approach and interaction with citizens in order to avoid insularity which might in turn pose risks to democracy.

The situation for refugees is quite different though. They connect to social media sites mainly in order to communicate with family, friends and influential figures in their social media networks. Their relationships in social media networks are not restricted to kinship and friendship only, but are weaved by pragmatic (material and cultural needs) and ideological factors, often the reasons that have driven them away from their homeland. The spaces of social media 
discussion and debate among refugees tend to be fractious, intensely politicized and polarized with users constantly commuting between open/public and private/closed Facebook spaces [2]. Such polarization can often lead to undesirable effects both in the countries the groups are located but to their homeland too. Thus, there is a need for secure, trusted environments where privacy of the users / participants in debates is respected and access is controlled to avoid insularity and its ill effects..

\section{Blockchain TeChNOLOGY AND TRUST}

Blockchain technology is a distributed network of transactions, including tracked changes, where the control of confirming some transactions is via public-private key cryptography. Thus, the blockchain is a trusted, shared public ledger that is open to inspection by everyone, but which cannot be controlled by any single entity. Blockchain operates on the principle that new transactions are broadcast to all nodes and that each node collects new transactions into a block. Each node in turn verifies the validity of the block before being able to transmit it further into the network [18, 19 and 20]

Therefore, Blockchain offers a self-controlling network that ensures anonymity, offers security and promotes trust at a very high level. The key concept is that Blockchain technology is designed as a decentralized peer-to-peer network. This does not rely on a central authority. Instead using a broadcast network transactions and blocks are broadcasted across the network using nodes. These are computers interconnected to the ad-hoc network that allows synchronization with other nodes No node knows a priori which version of the ledger (block of transactions) is valid, and to secure the blockchain against attacks, the cryptocurrency network relies on precise algorithms and consensus mechanisms. The most well-known of these are the PoW in the Bitcoin network and the proof of stake (PoS) in the Nxt network [18 and 21].

It is this lack of central controlling authority and the need to constantly validate transactions that makes such networks secure and trustworthy. Blockchain technology has developed a notorious reputation due to bad publicity relating to cryptocurrencies. However, it is these applications that make blockchain trustworthy and open. To transact without a trusted party, transactions must be publicly announced. This is where the main difference from traditional banking systems occurs. Conventional banking systems, in order to protect our money, require us to surrender our privacy. Thus, the customer trust to such systems is dependent on the integrity of a central authority and there are lots of cases that such trust has been breached repeatedly [18 and 20].

Blockchain technology has been around for a few decades in different formats, but it is still maturing and there are many concerns about scalability, costs, and security to be overcome before blockchain technology moves to widespread usage. The evolution though is very rapid and it is expected to be used to deliver social benefits and efficiency that can have widespread impacts in various aspects of life such as social interaction, medical systems (medical record mobility) and support of human life and integrity [20 and 21]. It is in such a context that the technology is introduced in a conceptual model of a system that combines blockchain with recommender systems to create a fully trusted social media environment that could support refuges, as shown in the next section of this work.

\section{TOWARDS A TRUSTED FORUM FOR DEMOCRCACY REGENARATION}

The diagram in fig 1 provides a schematic representation of the proposed work by the authors in developing a solution for secure and trusted political debate spaces (forums) within the context of social media.

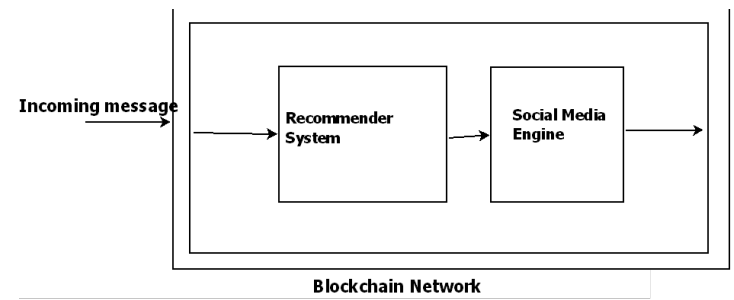

Fig. 1. A schematic representation of a trusted social network space

\section{A. Recommendations}

Trust and recommender systems in social media are important elements [22 and 23]. Furthermore, due to the factors that affect such a forum engine and to increase refugee participation, the use of a recommendation component is proposed. Thus, within the trusted forum engine the authors propose the integration of a component that will make recommendations about the following aspects:

1) People to people recommendation. This part will be used recommend people to people that share common characteristics. These characteristics include people of that are in the same or nearby area and have similar cultural characteristics.

2) Chat recommendation. This part will be used recommend who to chat with. In social media, a person could select the option to chat with different people, although it would be wiser in certain situations to chat with people that have similar needs and characteristics.

3) Resource recommendation. This part will be used to recommend resources to refugees. These will include things such as food, shelter and pharmacy. People new to a community will find particularly useful to be recommended information about resources.

4) News and event recommendation. This part will be used to recommend news and events to refugees. It is important for refugees that share common life aspects to read more personalized news and meet each other in person during various events.

5) Job recommendations. This part will be used to make relevant job recommendations to refugees. People who are forced to move between countries or to other countries due to difficult situations in their home country will benefit from such a tool. Moreover, such a tool might be found to be particularly useful at the early arrival or refugees that need casual jobs until they find something more permanent.

\section{B. Privacy and Trust}

Social media network applications suffer from privacy exposures, thus making it difficult for a user to submit data and receive more personalized recommendations [24, 25 and 26]. In addition, privacy needs to be protected within the 
forum to make it attractive to users, since privacy protection is important in both social media and recommender systems [25 and 27].

Privacy is considered as equally important as the other components of the system and appropriate privacy protection measures will be applied. More specifically, careful consideration about relevant method for privacy will be given to the recommendation components and to the user submitted messages to the system. The application of appropriate privacy protection measure will make users more confident in using the forum and make it a useful tool for them, thus making it easier for them finding relevant information and improving refugee lives.

The introduction of blockchain technology will enhance the trust element of such a setup. Users of the network will learn to operate through this transparent and at the same time safe system. While blockchain will allow them to view all transactions (messages in this systems) the use of recommender systems will guide them to process only the ones that are of interest to them. This will save considerable resources in terms of processing, will allow more successfully targeted transactions (exchange of messages) with other users of the network and all this under the protection of their anonymity [20 and 21].

This proposal is not unique in its social focus. The Finish government has had a very successful pilot scheme of managing refugee finance and movements based on blockchain technology. They have created cards that allow day-to-day transactions without the need of a bank account, whose establishment will meet with stumbling blocks due to their refugee status. At the same time the seamless records of these transactions allow for close monitoring of movements without the need to restrict their freedom of movement [28]. Although this is an excellent example of supporting physical activities, the authors believe that our proposal will provide a stepping stone for reaping the real benefits of the secure and trusted environment that such technologies offer.

\section{CONCLUSIONS AND FUTURE WORK}

The world is experiencing the unfortunate evolution of a growing mass of displaced people. Although survival is their first and primal concern, the need for peace and ability to live in a democratic environment is often the one that prevails once the basic needs are satisfied.

Political debate is in human nature and in the cases of refugees, that mostly have been displaced from their homeland due to political reasons, it is extremely important. To avoid risks and to minimize insularity, such debates should take place in controlled, secure and trusted environments. As most refugees are technologically savvy by necessity, social media are very familiar territories. The provision of secure spaces to setup their political forums in social media networks is a logical proposition. To this effect, the authors propose to explore the integration of recommender systems to meet this need.

The technical element of this work is still in progress. Due to the nature of the information to process in such systems, partnerships with researchers in countries where refugees originate from and those countries where they flee to have been established, to collect relevant data and allow for testing and evaluation in controlled environments.

Cost, efficiency and maturity of blockchain technology is going to be the major challenge but due to its involvement with the financial markets the evolution and improvements are expected to be rapid there.

\section{REFERENCES}

[1] Shat, F. and Pimenidis, E. (2017) Social Media and e-Voting - Secure and Trusted Political Forum for Palestine, in Jahankhani, H, et.al. (eds.) Global Security, Safety and Sustainability - The Security Challenges of the Connected World, CCIS 630, pp. 290-302

[2] Gillespie, M., Ampofo, L., Cheesman, M., Faith, B., Iliadou, E., Issa, A., Osseiran, S. and Skleparis, D. (2016) Mapping Refugee Media Journeys: Smartphones and Social Media Networks, Technical Report, DOI: 10.13140/RG.2.2.15633.22888

[3] Mousavi S.A.A., Pimenidis E, Jahankhani H. (2008) Cultivating Trust - An e-Government Development model for addressing the needs of developing countries, International Journal of Electronic Security and Digital Forensics (IJESDF), Vol. 1, No. 3, pp. 233-248.

[4] BBC 2016a. [online] http://www.bbc.co.uk/news/world-europe34131911/ (accessed 02/05/2016)

[5] Charmarkeh, H. (2013) Social Media Usage, Tahriib (Migration), and Settlement among Somali Refugees in France, Refuge, 29(1), 43-52.

[6] Axford, B. (2011) Talk About a Revolution: Social Media and the MENA Uprisings, Globalizations, 8(5), 681-686.

[7] Hampton, K., Inyoung, S. and Weixu L. (2017). Social Media and Political Discussion: When Online Presence Silences Offline Conversation. Information, Communication \& Society, 20(7), 10901107.

[8] UNCHR (2017) Europe Monthly Report - May 2017, UNCHR - The UN Refugee Agency.

http://data2.unhcr.org/en/situations/mediterranean [online] (accessed 10 July 2017)

[9] The Independent (2017) Grenfell Tower fire: First victim named as Syrian refugee Mohammed Alhajali [online] (accessed 10 July 2017)

[10] Kacem, A., Belkaroui, R., Jemal, D., Ghorbel, H., Faiz , R. and Abid, I.H.(2016) Towards collaborative e-government improvement using social media-based citizen's profile investigation, In Proceedings ICEGOV '15-16, March 01-03, 2016, Montevideo, Uruguay, DOI: http://dx.doi.org/10.1145/2910019.2910029

[11] Rainie, Lee, Duggan, M. "Privacy and Information Sharing" Pew Research Center, December 2015. Available at: http://www.pewinternet.org/2016/01/14/2016/Privacy-andInformation-Sharing/ (accessed 10 January 2017)

[12] Dincelli, E. and Goel, S. (2015) Research Design for Study of Cultural and Societal Influence on Online Privacy Behavior, In Proceedings of 2015 IFIP 8.11/11.13 Dewald Roode Information Security Research Workshop

[13] Trepte, S. \& Masur, P. K. (2016). Cultural differences in media use, privacy, and self-disclosure. Research report on a multicultural survey study. Germany: University of Hohenheim

[14] Unsworth, K. and Townes, A. (2012) Social Media and eGovernment: A case study assessing Twitter use in the implementation of the open government directive, In Proceedings of the American Society for Information Science and Technology January 2012, DOI: 10.1002/meet.14504901298

[15] Song, C. and Lee, S. (2016) Citizens' Use of Social Media in Government, Perceived Transparency, and Trust in Government, Public Performance \& Management Review, 39(2), 430-453, DOI: 10.1080/15309576.2015.1108798

[16] Dhir A, Torsheim T, Pallesen S and Andreassen CS (2017) Do Online Privacy Concerns Predict Selfie Behavior among Adolescents, Young Adults and Adults? Front. Psychol. 8:815

[17] Hamajoda A (2016) Embracing New Media in Political Communication: A Survey of Parliamentarians Attitudes and Practices in a Changing Media Landscape in West Africa. J Mass Communicat Journalism 6: 310 .

[18] Huckle, S. and White, M. (2016) Socialism and the Blockchain. Future Internet 2016, 8, 49, DOI: 10.3390/fi8040049

[19] Feig, E. (2018) A Framework for Blockchain-Based Applications, https://arxiv.org/abs/1803.00892

[20] Kewell, B., Adams, R. and Parry, G. (2017) Blockchain for good? Strategic Change: Briefings in Entrepreneurial Finance, 26 (5). pp. 429-437 
[21] Cocco, L., Pinna, A., and Marchesi, M. (2017) Banking on Blockchain: Costs Savings Thanks to the Blockchain Technology. Future Internet, 9, 25, DOI: 10.3390/fi9030025

[22] Zhou, X., Xu, Y., Li, Y., Josang, A., \& Cox, C. (2012). The state-ofthe-art in personalized recommender systems for social networking. Artificial Inteligence Review, 37(2), 119-132

[23] Guy, I., \& Geyer, W. (2014). Social recommender system tutorial. In Proceedings of the 8th ACM Conference on Recommender systems (pp. 403-404). ACM.

[24] Cutillo, L. A., Molva, R., \& Strufe, T. (2009). Safebook: A privacypreserving online social network leveraging on real-life trust. IEEE Communications Magazine, 47(12).

[25] Babu, K. S., Hota, J., \& Jena, S. K. (2014). Privacy preserving social networking. International Journal of Computational Science and Engineering, 9(3), 165-176.

[26] Zhou, B., \& Pei, J. (2008, April). Preserving privacy in social networks against neighborhood attacks. In Data Engineering, 2008. ICDE 2008. IEEE 24th International Conference on (pp. 506-515). IEEE.

[27] Polatidis, N., Georgiadis, C. K., Pimenidis, E., \& Stiakakis, E. (2017). Privacy-preserving recommendations in context-aware mobile environments. Information \& Computer Security, 25(1), 62-79.

[28] Orcutt, M., (2017) How Blockchain Is Kickstarting the Financial Lives of Refugees, MIT Technology Review https://www.technologyreview.com/s/608764/how-blockchain-iskickstarting-the-financial-lives-of-refugees/ (accessed 4 November 2018). 